\title{
Order and phase nucleation in nonequilibrium nanocomposite Fe- Pt thin films with perpendicular magnetic anisotropy
}

C. Clavero

William \& Mary

R. A. Lukaszew

William \& Mary, ralukaszew@wm.edu

J. M. Garcia-Martin

A. Cebollada

J. R. Skuza

William \& Mary

See next page for additional authors

Follow this and additional works at: https://scholarworks.wm.edu/aspubs

\section{Recommended Citation}

Clavero, C., Skuza, J. R., Garcia-Martin, J. M., Cebollada, A., Walko, D. A., \& Lukaszew, R. A. (2009). Order and phase nucleation in nonequilibrium nanocomposite Fe-Pt thin films with perpendicular magnetic anisotropy. Physical Review B, 79(10), 104436.

This Article is brought to you for free and open access by the Arts and Sciences at W\&M ScholarWorks. It has been accepted for inclusion in Arts \& Sciences Articles by an authorized administrator of W\&M ScholarWorks. For more information, please contact scholarworks@wm.edu. 


\section{Authors}

C. Clavero, R. A. Lukaszew, J. M. Garcia-Martin, A. Cebollada, J. R. Skuza, and D. A. Walko 


\title{
Order and phase nucleation in nonequilibrium nanocomposite Fe-Pt thin films with perpendicular magnetic anisotropy
}

\author{
C. Clavero, ${ }^{1,2, *}$ J. R. Skuza, ${ }^{3}$ J. M. García-Martín, ${ }^{2}$ A. Cebollada, ${ }^{2}$ D. A. Walko, ${ }^{4}$ and R. A. Lukaszew ${ }^{1,3}$ \\ ${ }^{1}$ Department of Applied Science, College of William and Mary, Williamsburg, Virginia 23187, USA \\ ${ }^{2}$ Instituto de Microelectrónica de Madrid, IMM (CNM-CSIC), Tres Cantos, Madrid 28760, Spain \\ ${ }^{3}$ Department of Physics, College of William and Mary, Williamsburg, Virginia 23187, USA \\ ${ }^{4}$ Advanced Photon Source, Argonne National Laboratory, Argonne, Illinois 60439, USA
}

(Received 10 December 2008; revised manuscript received 24 February 2009; published 31 March 2009)

\begin{abstract}
We report on the time evolution of mass transport upon annealing nonequilibrium Fe-Pt nanocomposite films, leading to nucleation of $L 1_{0}$ chemically ordered phase. The nonequilibrium nanocomposite films were fabricated by applying $\mathrm{Fe}^{+}$ion implantation to epitaxial $\mathrm{Pt}$ films grown on (001) $\mathrm{MgO}$ substrates, yielding $\mathrm{Fe}$ nanoclusters embedded in a Pt matrix at a tailored penetration depth. Time-resolved $\mathrm{x}$-ray diffraction studies were carried out using synchrotron radiation, allowing determination of the activation energy for nucleation of the FePt $L 1_{0}$ phase within the segregated nanoclusters during annealing. The growth of the segregated $L 1_{0}$ ordered phase was modeled using ideal grain-size law and found to be dominated by strain-driven surface nucleation. The activation energies were found to correlate with the nanocluster size. Magnetic characterization of selected annealed samples indicates perpendicular magnetic anisotropy with high coercive field coincident with high value of the chemical order parameter of the ordered phase within the magnetic nanoclusters.
\end{abstract}

DOI: 10.1103/PhysRevB.79.104436

PACS number(s): 75.75.+a, 75.70.Ak, 78.67.-n

\section{INTRODUCTION}

Nanocomposite materials with tailored properties are of interest for specific applications, and therefore it is important to understand the main processes involved during their synthesis. An important case to consider is nanocomposite thin films where the materials in the composite can form a binary alloy. These alloys often exhibit interesting phase diagrams, where some of the phases can have chemical order that in turn may affect physical properties in the material, as it is, for example, the case of the FePd and FePt alloys. ${ }^{1}$ In the latter systems, the chemical order also induces important changes in the magnetic anisotropy, and therefore these materials have potential applications, e.g., next generation magnetic media when in thin-film form. ${ }^{2-5}$ In general the phase behavior of such binary $A-B$ system is determined by a balance between the interactions $A A, B B$, and $A B$ which are typically of the order of $1 \mathrm{eV} .^{3}$ Nevertheless, in some cases the resulting effective pair interactions can be significantly smaller and can compete with thermal excitations in the system. In the Fe-Pt system a balance of three different types of energies determines the stability of a specific phase: (a) the formation enthalpy of the ordered structures, (b) the mixing enthalpy of the random alloy, and (c) the strain energy from atomic size mismatch. ${ }^{4}$ Of particular interest is the study of phase nucleation and ordering in nonequilibrium nanocomposite thin films consisting of nanometer-sized clusters or $\mathrm{Fe}$ particles embedded at a specific penetration depth within a $\mathrm{Pt}$ matrix.

One possible method to achieve nonequilibrium nanocomposite films is to utilize ion beams. In fact, ion implantation has been extensively used to modify the properties of materials, where the energy of the ions and the dose can be tailored to achieve specific properties. ${ }^{5}$ Very recently the use of low-energy self-ions demonstrated surface modification with creation of pans and mesas of dimensions up to $10 \mu \mathrm{m}$ on the surfaces of metallic crystals ${ }^{6}$ indicating that there is significant potential to use ion beams to modify materials in a useful way. In the case of nanocomposite thin films the dose can be selected to tailor the average nanocluster size and intercluster distance while the energy can be tuned to select the penetration depth of the clusters into the matrix. ${ }^{7}$ Specific to Fe-Pt, White et al. ${ }^{8,9}$ considered the implantation of $\mathrm{Fe}$ and $\mathrm{Pt}$ ions into $\mathrm{Al}_{2} \mathrm{O}_{3}$ and $\mathrm{SiO}_{2}$ matrices, followed by thermal annealing at $1100{ }^{\circ} \mathrm{C}$ to synthesize $2-10 \mathrm{~nm}$ nanoparticles with multiple crystalline orientations where a few exhibited perpendicular magnetic anisotropy. Additional reports indicate that annealing, ${ }^{10}$ rapid thermal annealing (RTA), ${ }^{11}$ and $\mathrm{x}$-ray rapid thermal annealing (XRTA) (Ref. 12) promote chemical ordering in stoichiometric FePt thin films.

Here, we have applied ion beams to obtain a segregated nonequilibrium nanocomposite thin film of Fe clusters implanted within a specific penetration depth into a Pt matrix where subsequent annealing treatments lead to a nanocomposite material with desired magnetic properties, i.e., decoupled nanoclusters with large perpendicular magnetic anisotropy embedded in a nonmagnetic Pt matrix. We have used synchrotron radiation in order to track the microstructure real-time evolution of the films under annealing. We find that the subsequent annealing treatments after implantation give rise to highly oriented and chemically ordered $L 1_{0}$ FePt crystallites with large magnetic anisotropy where the $c$ axis is determined by the Pt matrix crystal structure.

\section{EXPERIMENTAL APPROACH}

Epitaxial 30-nm-thick Pt films were grown at room temperature (RT) on $\mathrm{MgO}(001)$ substrates by dc triode sputtering in a system working in ultrahigh vacuum (UHV) conditions (background pressure of $5 \times 10^{-9}$ Torr). X-ray diffraction (XRD) scans were performed on selected films 
using a standard four-circle diffractometer with $\mathrm{Cu} K_{\alpha}$ radiation $(\lambda=1.5418 \AA)$ in the Bragg-Brentano configuration and with $\frac{1}{4}$ degree slits. Subsequently, the films were implanted using $30 \mathrm{keV} \mathrm{Fe} \mathrm{singly-charged} \mathrm{ions} \mathrm{in} \mathrm{a} \mathrm{heavy-ion} \mathrm{accel-}$ erator described elsewhere. ${ }^{13}$ Ion implantation was carried out at RT and at a pressure of $10^{-6}$ Torr. The implantation conditions were chosen after stopping and range of ions in matter simulations ${ }^{14,15}$ were carried out to estimate the optimal conditions for 1:1 stoichiometry at a mean projected depth of $8 \mathrm{~nm}$ from the film surface. Implanted doses were in the $10^{16}$ ions $/ \mathrm{cm}^{2}$ range with typical ion-implanting current density $\sim 200 \mathrm{nA} / \mathrm{cm}^{2}$.

In order to investigate the mass-transport kinetics for the FePt alloy formation and to determine a suitable temperature, time, and activation energy required to nucleate a specific phase, e.g., chemically ordered $L 1_{0}$ FePt clusters, sequential isothermal annealing treatments and XRD experiments using $9.2 \mathrm{keV}$ synchrotron radiation were performed at the Sector 7 beamline of the Advanced Photon Source. The samples were mounted inside a high-vacuum chamber (base pressure of $\sim 3.0 \times 10^{-7}$ Torr) equipped with a resistive heating oven and provided with a Be dome to allow x-ray penetration. The annealing temperature was monitored via multiple thermocouples as well as the angular position of the $\mathrm{MgO}(004)$ reflection using the known thermal-expansion coefficient of MgO. ${ }^{16}$ Simultaneously, isothermal XRD scans were carried out in real time in the Bragg-Brentano geometry.

Finally, selected samples with different $\mathrm{Fe}^{+}$implanted doses were annealed at $450{ }^{\circ} \mathrm{C}$ for different time periods ranging from $1 \mathrm{~min}$ to several hours in an UHV chamber (base pressure of $\sim 5 \times 10^{-9}$ Torr) to investigate the correlation between cluster microstructure and magnetic anisotropy. The magnetic anisotropy was studied ex situ by measuring polar Kerr hysteresis loops using $2.3 \mathrm{eV}$ radiation and perpendicular magnetic fields of up to $1.5 \mathrm{~T}$ before and after annealing the sample. Magnetic force microscopy (MFM) images mapping the magnetic poles at the sample surface were also obtained after a demagnetization process with the magnetic field applied along the perpendicular direction.

\section{A. Activation energy determination using synchrotron radiation}

For this study, the epitaxial Pt thin-film samples were implanted with a $\mathrm{Fe}^{+}$dose of $\sim 3 \times 10^{16}$ ions $/ \mathrm{cm}^{2}$ at $30 \mathrm{keV}$. The superlattice $\mathrm{FePt}(001)$ reflection, characteristic of the $L 1_{0}$ ordered phase, was tracked in real time for each annealing temperature in the range of $400-600{ }^{\circ} \mathrm{C}$. We found that mass transport leading to the onset of the $\mathrm{FePt}(001)$ reflection occurs at moderate temperature (i.e., around $450{ }^{\circ} \mathrm{C}$ ). We notice that this temperature is well below the $1100{ }^{\circ} \mathrm{C}$ reported for clusters embedded in dielectric matrices. ${ }^{9}$ We also find that the ordered-phase nucleates with the $c$ axis following the crystallography of the Pt matrix, i.e., in (111) films the $c$ axis of the ordered $L 1_{0}$ FePt clusters maintains a similar orientation with respect to the film crystallographic directions rather than being parallel to the surface normal as the case in (001)-oriented films. The time evolution of the signature peaks under annealing was investigated to establish

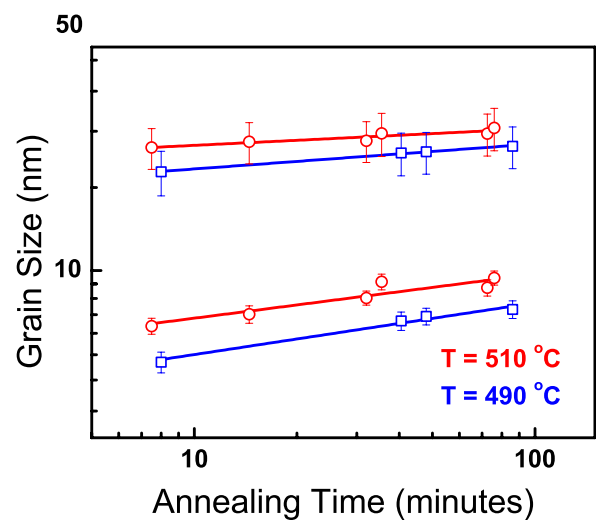

FIG. 1. (Color online) Time-dependent grain-size evolution upon two annealing temperatures $\left(490\right.$ and $\left.510^{\circ} \mathrm{C}\right)$. Two grain sizes are found at both temperatures; the upper two curves correspond to the larger grain size $(d>20 \mathrm{~nm})$, whereas the lower two curves correspond to the smaller grain size $(d<10 \mathrm{~nm})$. A larger grain growth exponent [Eq. (1)] $n \approx 15$ is found for the larger grain size, whereas the smaller grain size exhibits $n \approx 6$.

scaling laws for coarsening of the ordered nanoclusters with time. The activation energy could be estimated from the time evolution of these isothermal scans as discussed below. A Gaussian fitting of the $\mathrm{FePt}(001)$ reflections revealed two typical average nanocluster sizes since two Gaussian curves were needed to fit the peaks plus an additional broad Gaussian for the background, the latter is probably due to thermal diffuse scattering. The average grain size $d$ of the $L 1_{0} \mathrm{FePt}$ nanoclusters was calculated from the full width at half maximum (FWHM) of the Gaussian curves using the Scherrer equation. ${ }^{17}$ Figure 1 depicts the evolution of the nanoclusters grain size as a function of the annealing time for two different annealing temperatures, namely, 490 and $510{ }^{\circ} \mathrm{C}$. As can be observed, both grain-size populations increase their size as the annealing time increases. It is worth noticing that the Scherrer equation does not take into account the broadening of the peaks associated with the strain present in the system. Nevertheless, this contribution was estimated to be around $1.5 \%$ in the grain size for the smaller grains $(d<10 \mathrm{~nm})$ and well below $10 \%$ for the larger grains size $(d>20 \mathrm{~nm})$. The corresponding error bars were added to Fig. 1.

Internal interfaces may evolve to minimize surface energy via grain growth or Ostwald ripening. ${ }^{18}$ Alternately, they may move due to a volumetric change such as recrystallization or a phase transition. In this case we observe the nucleation of a different phase (FePt $\left.L 1_{0}\right)$ that grows under annealing. We have used the Burke and Turnbull ${ }^{19}(\mathrm{~B}-\mathrm{T})$ model to explain the growth of the $L 1_{0}$ FePt nanoclusters. In the isothermal B-T model it is assumed that grain growth is caused by the movement of grain boundaries and atom transport across these boundaries ${ }^{20}$ where the driving pressure on a grain boundary arises from its curvature. The evolution of the average $L 1_{0}$ FePt grain size $d$ shown in Fig. 1 yields a similar form to B-T's equation for the kinetics of grain growth,

$$
d=k t^{n^{-1}}
$$

where $n$ is the grain growth exponent and $k$ is the grain growth parameter. In the ideal B-T model, $n=2$, but many 
experimental results have shown that $n$ can be larger than 2 and varies with composition and temperature. ${ }^{21}$ The growth of the $L 1_{0}$ FePt nanoclusters is also a thermally activated process where the average grain size $d$ increases with the annealing temperature $T$. Thus, the grain growth rate parameter $k$ can be written as

$$
k=k_{0} e^{-E_{G^{\prime}} / k T},
$$

where $k_{B}$ is the Boltzmann constant, $T$ is the temperature in $\mathrm{K}, k_{0}$ is a constant, and $E_{G}$ is the activation energy for grain growth. Our studies reveal that the typical activation energies for grain growth in our samples with grain sizes less than 10 $\mathrm{nm}$ is $\sim 0.3 \pm 0.1 \mathrm{eV}$, while for larger sizes it is $\sim 0.5 \pm 0.1 \mathrm{eV}$. We also find that the grain growth exponent $n$ has a typical value of around 5-6 for smaller grains $(d$ $<10 \mathrm{~nm})$ while it was much larger $(n=15)$ for larger grains $(d>20 \mathrm{~nm})$, indicating different growth kinetics. As mentioned above, grains can also grow due to Ostwald ripening Ref. 18. In this process atoms detached from small clusters of the newly formed phase must diffuse through the matrix to attach to large clusters of the same phase so that large grains grow at the expense of small particles. Thus, the interfacial energy between the matrix and the new phase is reduced. The grain growth exponent for Ostwald ripening of particles in polycrystalline materials can be as large as $n=4$ or 5 in the case of grain-boundary diffusion controlled grain growth, ${ }^{22}$ and in the case where grain growth is controlled by surface nucleation, the value of $n$ can be randomly large. ${ }^{23}$ Thus, we postulate that our values for $n$ are controlled by surface nucleation and Ostwald ripening. ${ }^{20}$ The main driving force for surface nucleation is the interfacial strain between the $\mathrm{Fe}$ clusters and the Pt matrix. Evidence of strain in the Pt matrix will be discussed further in Sec. II B. It is worth commenting here that many kinetic and thermodynamic investigations on near stoichiometric bulk and thin-film FePt samples have been reported in the literature ${ }^{20,24,25}$ using techniques such as differential scanning calorimetry, XRD, and transmission electron microscopy to extract activation energies for the formation of different phases (e.g., $A 1$ to $L 1_{0}$ ), and models such as the Johnson-Mehl-Avrami-Kolmogorov formulation ${ }^{20}$ and saturation-state growth rate model ${ }^{25}$ were used. For example, typical values reported for activation energies in bulk FePt alloys using differential scanning calorimetry are in the vicinity of 1-2 eV (Ref. 26) while in the case of FePt thin films grown by dc magnetron sputtering from a stoichiometric FePt target, Li et al. ${ }^{25}$ found activation energies around 0.9 $\mathrm{eV}$. Thus, our smaller values indicate that the activation energy for nucleation of the ordered phase in the nanocomposite films investigated may be dominated by strain-induced surface mass-transport mechanisms, particularly noticeable in smaller clusters. Typically surface dominated mass transport leads to smaller activation energy than bulk dominated processes $^{28}$ and hence lower temperature for the onset of the nucleation of the $L 1_{0}$ phase.

\section{B. Microstructure and magnetic anisotropy study}

It is well known that the FePt $L 1_{0}$ ordered-phase lattice distortion leads to high magnetic anisotropy along the $c$ axis

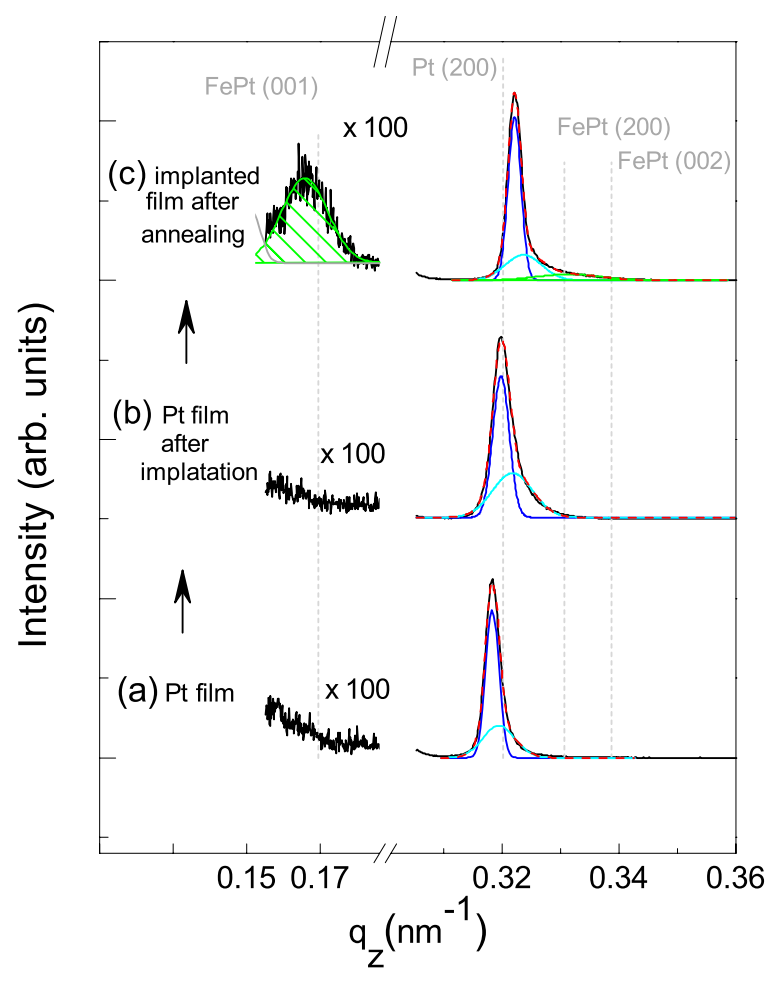

FIG. 2. (Color online) XRD symmetric scans for (a) Pt film as deposited, (b) $\mathrm{Pt}$ film after $\mathrm{Fe}$ ion implantation (dose:5 $\times 10^{16}$ ions $/ \mathrm{cm}^{2}$ ), and (c) Fe-implanted Pt film after $1 \mathrm{~h}$ annealing at $450{ }^{\circ} \mathrm{C}$. The onset of $L 1_{0}$ FePt ordered phase after annealing is evidenced by the fundamental $\operatorname{FePt}(002)$ and the superlattice $\mathrm{FePt}(001)$ reflections. A Gaussian fitting of the reflections is shown, with the Pt film reflections being represented by blue lines, the ordered FePt phase with green lines, and the cumulative fitting with red lines.

and constitutes a characteristic signature of its formation. In the present case the correlation between microstructure as determined from XRD and the magnetic anisotropy was investigated before and after annealing treatments with magneto-optical Kerr hysteresis loops, measured both in polar and transverse geometries after tailoring the $\mathrm{Fe}^{+}$implanted dose and the annealing temperature in selected samples. Complementary, MFM images mapped the magnetic poles at the sample surface after a demagnetization process with the magnetic field applied along the perpendicular direction.

For this study, epitaxial 30-nm-thick Pt (001) films [Fig. 2(a)] were implanted with two different $\mathrm{Fe}^{+}$doses and two typical samples will be presented; sample A was implanted with a $\mathrm{Fe}^{+}$dose of $5 \times 10^{16}$ ions $/ \mathrm{cm}^{2}$ whereas half this dose was applied in sample B in order to investigate the early stages of nanocluster formation and magnetic coupling. Subsequently to the implantation the films were annealed at $450{ }^{\circ} \mathrm{C}$ [a value determined from our previous studies, well below the reported FePt bulk order-disorder temperature $\sim 1300{ }^{\circ} \mathrm{C}$ (Ref. 29)] for different periods of time ranging from $1 \mathrm{~min}$ to several hours under ultrahigh vacuum conditions (base pressure of $\sim 5 \times 10^{-9}$ Torr).

XRD scans obtained for sample A reveal broadening and an overall shift of the $\mathrm{Pt}(200)$ reflection toward higher angles 
after Fe ion implantation [Fig. 2(b)], evidencing a decrease in the crystalline grain size and contraction of the lattice, i.e., evidence of strain in the Pt matrix. No trace of crystalline Fe was found despite of the large amount of $\mathrm{Fe}$ ions implanted, signifying a random distribution of highly disordered $\mathrm{Fe}$ nanoclusters. To favor the migration of the intervening atoms toward formation of FePt ordered alloy nanoclusters, sample A was UHV annealed for $1 \mathrm{~h}$ at $450{ }^{\circ} \mathrm{C}$. The onset of chemically ordered $L 1_{0}$ phase was evidenced in the XRD scans [Fig. 2(c)] by the appearance of the superlattice reflection (001), corresponding to the doubled periodicity in the unit cell. From the position of the $\mathrm{FePt}(001)$ symmetric and the $\mathrm{FePt}(220)$ asymmetric reflections, perpendicular $(c)$ and an in-plane (a) lattice parameters of $3.783 \pm 0.001$ and $3.904 \pm 0.001 \AA$, respectively, were calculated. Thus, a tetragonal distortion characteristic of the $L 1_{0}$ chemically ordered phase is observed in the ordered FePt phase with a $c / a$ ratio of $0.969 \pm 0.001$. From the FWHM of the symmetric $\mathrm{FePt}(001)$ reflection it was inferred a vertical grain size of $4.10 \pm 0.06 \mathrm{~nm}$ for the FePt ordered phase. It is worth noticing that the epitaxial relationship of the FePt nanocrystallites with the substrate is the same as the predominant one found between the $\mathrm{Pt}$ film and the substrate, namely, $[010](001) \mathrm{FePt} / /[010](001) \mathrm{MgO}$. This result corroborates that the Pt lattice determines the direction of growth of the FePt alloy clusters and gives rise to highly oriented $\mathrm{FePt}(001)$ nanocrystallites.

In order to correlate the microstructure with the magnetic anisotropy achieved after sequential implantation and annealing processes, a complete magnetic characterization was carried out on sample A. In Fig. 3, polar (upper row) and transverse (lower row) Kerr hysteresis loops measured on this sample before and after annealing at $450{ }^{\circ} \mathrm{C}$ for $1 \mathrm{~h}$ are shown. Before annealing [Fig. 3(a)], clear in-plane magnetic anisotropy is observed, demonstrated by in-plane coercivity $(60 \mathrm{Oe})$ and large remanence $(92 \%)$, along with large perpendicular saturation field $(15 \mathrm{kOe})$. These characteristics are consistent with the presence of disordered $\mathrm{Fe}$ small clusters inside the Pt film, although large enough to overcome superparamagnetic behavior at RT [ $\sim 25 \mathrm{~nm}$ for Fe (Ref. 30) considering spherical clusters]. Moreover, the squareness of the in-plane loop suggests strong coupling between the Fe clusters since isolated clusters would not produce a sharp transition but rather a smoother one due to the distribution of individual coercive fields. Such coupling can be attributed to polarized $\mathrm{Pt}$ at the interface and between adjacent Fe clusters that acts as exchange transmitter. ${ }^{31}$ These collective behavior is corroborated by MFM experiments (bottom row, left) indicating that the sample exhibited magnetic domains with in-plane magnetization that were locally modified by a magnetically harder MFM tip after consecutive scans because of the soft magnetic nature of the sample..$^{32}$ An important magnetic anisotropy change is observed in the implanted film after annealing at $450{ }^{\circ} \mathrm{C}$ for $1 \mathrm{~h}$ [Fig. 3(b)] where remarkable increase in the perpendicular coercivity $(2.7 \mathrm{kOe})$ and remanence are observed, along with a large in-plane saturation field value $(10 \mathrm{kOe})$, evidencing the onset of perpendicular magnetic anisotropy in the system, compatible with the XRD observation of FePt $L 1_{0}$ ordered phase after annealing. It is worth noticing that the shape of the perpendicular a) before annealing

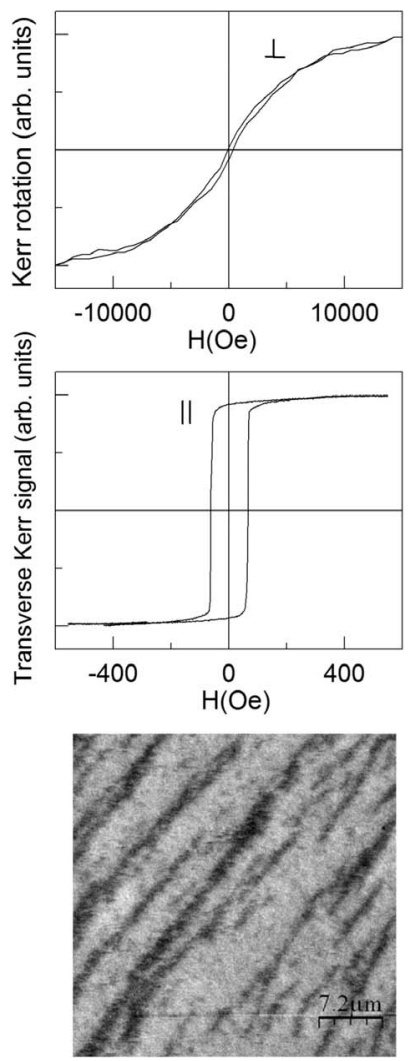

b) after annealing

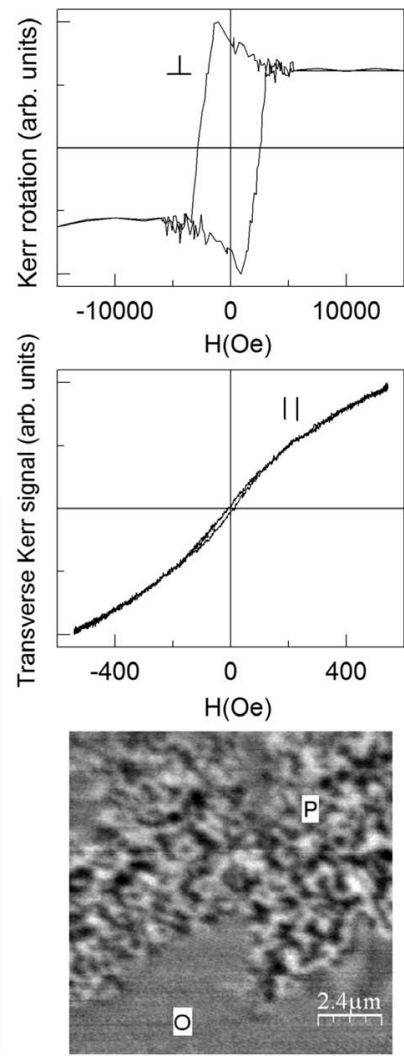

FIG. 3. Polar and transverse Kerr loops along with MFM images for the Fe-implanted (dose: $5 \times 10^{16}$ ions $/ \mathrm{cm}^{2}$ ) Pt film (sample A) (a) before and (b) after annealing at $450{ }^{\circ} \mathrm{C}$ for $1 \mathrm{~h}$. The perpendicular loop measured after annealing shows the inhomogeneity of the sample, where areas with opposite magneto-optical constants are illuminated. MFM images before and after annealing (please notice their different scale) show the anisotropy change and the onset of domains with perpendicular magnetization for the latter in the region labeled as $\mathrm{P}$, while the region without perpendicular magnetization is labeled $\mathrm{O}$.

loop after annealing evidences the somewhat inhomogeneous nature of this sample. Polar Kerr spectra measured with smaller spot size sampling different areas revealed opposite values of the polar Kerr rotation due to differences in ordering degree and interparticle coupling. The resultant composition of various such areas in the sample gives rise to the loop shape shown in Fig. 3(b). MFM observations [Fig. 3(b),bottom] corroborate the inhomogeneity of the sample since regions with perpendicular magnetic anisotropy (area labeled P) with black and white contrast due to opposite magnetic poles at the sample surface are observed ${ }^{33}$ whereas outside (area labeled $\mathrm{O}$ ) there is no magnetic contrast. It is worth noticing that consecutive scans did not show any significant change because the sample coercivity is now several times larger than the tip stray field. The observed inhomogeneity is attributed to nonuniform ion beam and the high $\mathrm{Fe}^{+}$ ion dose applied to sample A $\left(5 \times 10^{16}\right.$ ions $\left./ \mathrm{cm}^{2}\right)$ and consequent charge effects leading to inhomogeneous implanted regions. It is worth noticing that this inhomogeneity was not detected before annealing due to the soft in-plane magnetic 


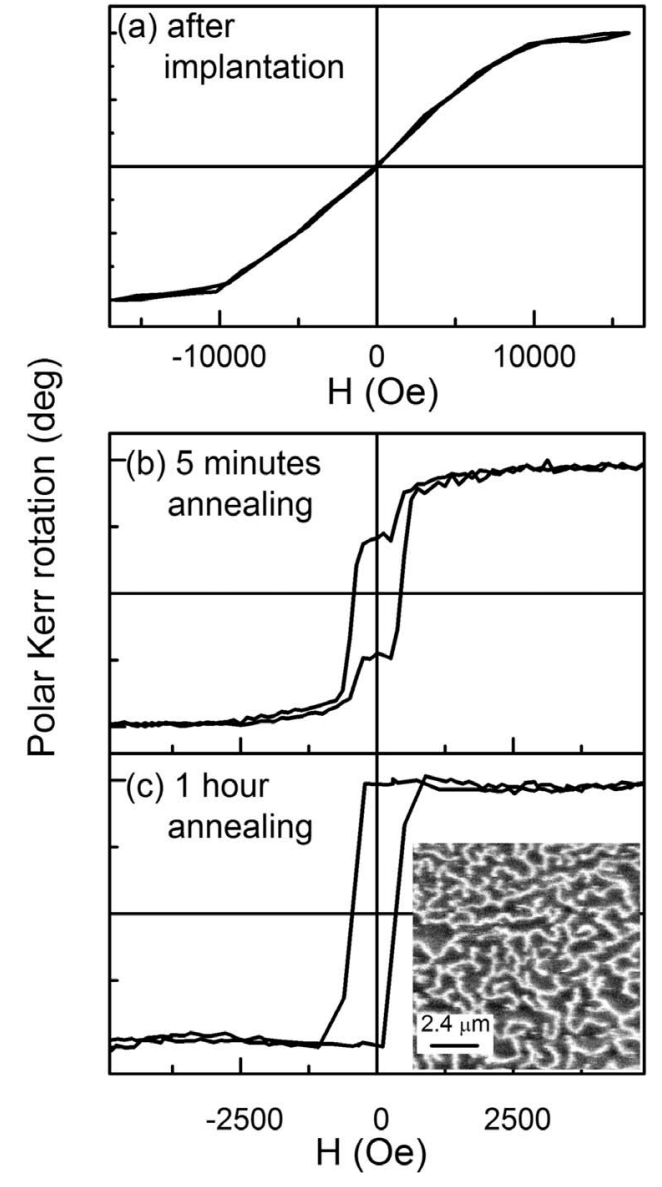

FIG. 4. Polar Kerr loops for the Fe-implanted (dose:2.5 $\times 10^{16}$ ions $/ \mathrm{cm}^{2}$ ) Pt film (sample B) (a) after implantation and (b) after $5 \mathrm{~min}$ annealing at $450{ }^{\circ} \mathrm{C}$. At this stage the sample exhibits magnetically decoupled nanoregions with strong perpendicular magnetic anisotropy. (c) After additional $8 \mathrm{~min}$ (13 min total) annealing at $450{ }^{\circ} \mathrm{C}$. The inset to (c) depicts a MFM image of such sample after the two annealings, showing a homogeneous mazelike domain pattern on the surface indicating magnetic coupling.

anisotropy observed with significantly larger magnetic domains. Further, this inhomogeneity cannot be attributed to differential mass transport of $\mathrm{Fe}$ atoms within the Pt film since their diffusion coefficient at the annealing temperature $\left[\sim 0.2 \mathrm{~nm}^{2} / \mathrm{s}\right.$ (Ref. 34)] leads to diffusion lengths several orders of magnitude shorter than the observed inhomogeneities.

In order to further investigate the magnetic coupling between clusters during early stages of nucleation of the ordered phase, it was necessary to improve the homogeneity in the implanted area. Therefore a second Pt film grown under identical conditions (sample B) was implanted with half the dose, namely, $2.5 \times 10^{16}$ ions $/ \mathrm{cm}^{2}$. No perpendicular magnetic anisotropy was detected before annealing, demonstrated by the polar Kerr loop shown in Fig. 4(a). In this case, the annealing time was also reduced to monitor the early stages of formation for the ordered phase. A short 5 min annealing was carried out under UHV conditions at $450{ }^{\circ} \mathrm{C}$. Due to the small amount of Fe implanted, no trace of ordered phases was found in the XRD scans after implan- tation, but the polar Kerr loop shown in Fig. 4(b) demonstrates the onset of perpendicular magnetic anisotropy related to the appearance of the FePt $L 1_{0}$ phase. At this point the film exhibits a "two-shoulder" hysteresis loop, attributed to the superposition of two phases, both with perpendicular magnetic anisotropies but with different coercive and saturation fields. This behavior is characteristic of two different $\mathrm{FePt}$ ordered phases with different $L 1_{0}$ ordering degree present in the film and with little or no exchange coupling between them, as has also been reported previously. ${ }^{35,36}$ To achieve a single ordered phase, a second UHV annealing at $450{ }^{\circ} \mathrm{C}$ was carried out during $8 \mathrm{~min}$. As can be observed in Fig. 4(c), only the phase with higher coercive field (400 Oe) and $100 \%$ remanence prevails, corresponding to a phase with higher degree of chemical order and also illustrating that the growth of this phase evolved from Ostwald ripening. MFM images after such second annealing show the characteristic maze pattern of $L 1_{0}$ thin films, ${ }^{37}$ indicating that there is magnetic coupling between clusters and good homogeneity since only regions with perpendicular magnetization are observed.

\section{CONCLUSIONS}

In summary, we have investigated time evolution of mass transport leading to binary-alloy nucleation and ordering (i.e., $L 1_{0}$ phase) in far-from-equilibrium nanocomposite Fe-Pt films. The films consisted of highly anisotropic FePt nanoclusters embedded on a Pt matrix obtained after implanting $30 \mathrm{keV} \mathrm{Fe} \mathrm{singly-charged} \mathrm{ions} \mathrm{on} \mathrm{Pt} \mathrm{films,} \mathrm{and} \mathrm{sub-}$ sequently annealing at moderate temperature during different time periods. Mass-transport mechanisms during nucleation of the ordered phase were investigated in real time using synchrotron radiation and found to be dominated by surface diffusion mechanisms driven by interfacial strain. The activation energy found for the formation of the ordered phase $(0.3-0.5 \mathrm{eV})$ depends on the cluster size and is smaller than that reported for bulk and stoichiometric thin-film samples leading to lower temperature for nucleation of the $L 1_{0}$ phase. To test our understanding of the correlated anisotropymicrostructure properties studies, specific implantation and annealing conditions were applied to selected samples leading to nanocomposite films with vertically ordered nanoclusters of $4 \mathrm{~nm}$ average grain size exhibiting strong perpendicular magnetic anisotropy and high coercivity. Upon annealing during $5 \mathrm{~min}$ at $450{ }^{\circ} \mathrm{C}$ a sample implanted with half the dose than the previous, the nanocluster regions appear magnetically decoupled, while increasing the annealing time promotes magnetic coupling. These findings may have important implications for magnetic media applications as well as for the design of other nanocomposites with materials that can form binary alloys.

\section{ACKNOWLEDGMENTS}

Funding from NSF (Grant No. DMR-0355171), Research Corporation Cottrell Scholar Award, and the American Chemical Society under Grant No. PRF-41319-AC10 is acknowledged. Funding from different Spanish Institutions, 
CM (Grant No. S-0505/MAT/0194) (NANOMAGNET) and MEC (Grant No. MAT2005-05524-C02-01), is also acknowledged. Use of the Advanced Photon Source was supported by the Office of Science, Office of Basic Energy Sci- ences, U.S. Department of Energy under Contract No. DEAC02-06CH11357. The authors also acknowledge R. Irving, M. Brown, and M. Mitra for assistance during ion implantation at the Toledo Heavy Ion Accelerator (THIA).
*Author to whom correspondence should be addressed; cclavero@wm.edu

${ }^{1}$ A. Cebollada, R. F. C. Farrow, and M. F. Toney, in Magnetic Nanostructures, edited by H. S. Nalwa (American Scientific, Los Angeles, 2002), pp. 93-118.

${ }^{2}$ D. Weller and A. Moser, IEEE Trans. Magn. 35, 4423 (1999).

${ }^{3}$ H. Reichert, A. Schöps, I. B. Ramsteiner, V. N. Bugaev, O. Shchyglo, A. Udyansky, H. Dosch, M. Asta, R. Drautz, and V. Honkimäki, Phys. Rev. Lett. 95, 235703 (2005).

${ }^{4}$ G. Bozzolo, R. A. Lukaszew, and J. E. Garces, Appl. Phys. Lett. 88, 011915 (2006).

${ }^{5}$ L. Ding, T. P. Chen, Y. Liu, M. Yang, J. I. Wong, K. Y. Liu, F. R. Zhu, and S. Fung, Nanotechnology 18, 455306 (2007).

${ }^{6}$ C. P. Flynn, M. Ondrejcek, and W. Swiech, J. Phys.: Condens. Matter 20, 395001 (2008)

${ }^{7}$ P. D. Townsend, J. C. Kelly, and N. E. W. Hartley, Ion Implantation, Sputtering and Their Applications (Academic, New York, 1976).

${ }^{8}$ C. W. White, S. P. Withrow, J. D. Budai, L. A. Boatner, K. D. Sorge, J. R. Thompson, K. S. Beaty, and A. Meldrum, Nucl. Instrum. Methods Phys. Res. B 191, 437 (2002).

${ }^{9}$ C. W. White, S. P. Withrow, K. D. Sorge, A. Meldrum, J. D. Budai, J. R. Thompson, and L. A. Boatner, J. Appl. Phys. 93, 5656 (2003).

${ }^{10}$ T. O. Seki, Y. K. Takahashi, and K. Hono, IEEE Trans. Magn. 41, 3799 (2005).

${ }^{11}$ T. Yokota, M. L. Yan, Yingfan Xu, L. Gao, R. Zhang, L. Nicholl, L. Yuan, R. Skomski, D. J. Sellmyer, S. H. Liou, Chih-Huang Lai, Cheng-Han Yang, and Sheng-Huang Huang, J. Appl. Phys. 97, $10 \mathrm{H} 306$ (2005).

${ }^{12}$ J. R. Skuza, R. A. Lukaszew, E. M. Dufresne, D. A. Walko, C. Clavero, A. Cebollada, C. N. Cionca, and R. Clarke, Appl. Phys. Lett. 90, 251901 (2007).

${ }^{13}$ R. R. Haar, D. J. Beideck, L. J. Curtis, T. J. Kvale, A. Sen, R. M. Schectman, and H. W. Stevens, Nucl. Instrum. Methods Phys. Res. B 79, 746 (1993).

${ }^{14}$ J. P. Biersack and L. Haggmark, Nucl. Instrum. Methods 174, 257 (1980)

${ }^{15}$ J. F. Ziegler, J. P. Biersack, and U. Littmark, The Stopping and Range of Ions in Solids, (Pergamon, New York, 1985).

${ }^{16}$ M. D. Biegalski, J. H. Haeni, S. Trolier-McKinstry, D. G. Schlom, C. D. Brandle, and A. J. Ven Graitis, J. Mater. Res. 20,
952 (2005).

${ }^{17}$ B. E. Warren, X-Ray Diffraction (Addison-Wesley, Reading, MA, 1969).

${ }^{18}$ R. El-Khozondar, H. El-Khozondar, G. Gottstein, and A. Rollet, Egypt. J. Solids 29, 35 (2006).

${ }^{19}$ J. E. Burke and D. Turnbull, Prog. Met. Phys. 3, 220 (1952).

${ }^{20}$ M. Moriyama, K. Matsunaga, and M. Murakami, J. Electron. Mater. 32, 261 (2003).

${ }^{21}$ F. J. Humphreys and M. Hatherly, Recrystallization and Related Annealing Phenomena (Pergamon, Oxford, 1995).

${ }^{22}$ A. J. Ardell, Acta Metall. 20, 601 (1972).

${ }^{23}$ S. V. Solomatov and D. J. Stevanson, J. Geophys. Res. 98, 5407 (1993).

${ }^{24}$ K. Barmak, J. Kim, S. Shell, E. B. Svedberg, and J. K. Howard, Appl. Phys. Lett. 80, 4268 (2002).

${ }^{25}$ X. H. Li, B. T. Liu, W. Li, H. Y. Sun, D. Q. Wu, and X. Y. Zhang, J. Appl. Phys. 101, 093911 (2007).

${ }^{26}$ D. C. Berry and K. Barmak, J. Appl. Phys. 101, 014905 (2007).

${ }^{28}$ R. A. Lukaszew, Y. Sheng, C. Uher, and R. Clarke, Appl. Phys. Lett. 76, 724 (2000).

${ }^{29}$ A. Cebollada, D. Weller, J. Sticht, G. R. Harp, R. F. C. Farrow, R. F. Marks, R. Savoy, and J. C. Scott, Phys. Rev. B 50, 3419 (1994).

${ }^{30}$ C. Martínez Boubeta, C. Clavero, J. M. García-Martín, G. Armelles, A. Cebollada, L1. Balcells, J. L. Menéndez, F. Peiró, A. Cornet, and Michael F. Toney, Phys. Rev. B 71, 014407 (2005).

${ }^{31}$ E. Navarro, Y. Huttel, C. Clavero, G. Armelles, and A. Cebollada, Appl. Phys. Lett. 84, 2139 (2004).

${ }^{32}$ J. M. Garcia, A. Thiaville, and J. Miltat, J. Magn. Magn. Mater. 249, 163 (2002).

${ }^{33}$ C. Clavero, J. M. García-Martín, J. L. Costa Kramer, G. Armelles, A. Cebollada, Y. Huttel, R. A. Lukaszew, and A. J. Kellock, Phys. Rev. B 73, 174405 (2006).

${ }^{34}$ Y. Endo, K. Oikawa, T. Miyazaki, O. Kitakami, and Y. Shimada, J. Appl. Phys. 94, 7222 (2003).

${ }^{35}$ J. P. Liu, Y. Liu, C. P. Luo, Z. S. Shan, and D. J. Sellmyer, J. Appl. Phys. 81, 5644 (1997).

${ }^{36}$ J. P. Liu, C. P. Luo, Y. Liu, and D. J. Sellmyer, Appl. Phys. Lett. 72, 483 (1998).

${ }^{37}$ V. Gehanno, A. Marty, B. Gilles, and Y. Samson, Phys. Rev. B 55, 12552 (1997). 